\title{
Top quark decays with flavor violation in the B-LSSM
}

\author{
Jin-Lei Yang ${ }^{1, a}$, Tai-Fu Feng ${ }^{1, b}$, Hai-Bin Zhang ${ }^{1, c}$, Guo-Zhu Ning ${ }^{1, d}$, Xiu-Yi Yang ${ }^{2}$ \\ ${ }^{1}$ Department of Physics, Hebei University, Baoding 071002, China \\ ${ }^{2}$ Department of Science, University of Science and Technology Liaoning, Anshan 114051, China
}

Received: 13 March 2018 / Accepted: 21 May 2018 / Published online: 1 June 2018

(c) The Author(s) 2018

\begin{abstract}
The decays of top quark $t \rightarrow c \gamma, t \rightarrow c g, t \rightarrow$ $c Z, t \rightarrow c h$ are extremely rare processes in the standard model (SM). The predictions on the corresponding branching ratios in the SM are too small to be detected in the future, hence any measurable signal for the processes at the LHC is a smoking gun for new physics. In the extension of minimal supersymmetric standard model with an additional local $U(1)_{B}-L$ gauge symmetry (B-LSSM), new gauge interaction and new flavor changing interaction affect the theoretical evaluations on corresponding branching ratios of those processes. In this work, we analyze those processes in the B-LSSM, under a minimal flavor violating assumption for the soft breaking terms. Considering the constraints from updated experimental data, the numerical results imply $B r(t \rightarrow c \gamma) \sim 5 \times 10^{-7}, B r(t \rightarrow c g) \sim 2 \times 10^{-6}$, $B r(t \rightarrow c Z) \sim 4 \times 10^{-7}$ and $\operatorname{Br}(t \rightarrow c h) \sim 3 \times 10^{-9}$ in our chosen parameter space. Simultaneously, new gauge coupling constants $g_{B}, g_{Y B}$ in the B-LSSM can also affect the numerical results of $\operatorname{Br}(t \rightarrow c \gamma, c g, c Z, c h)$.
\end{abstract}

Keywords Rare decay · B-LSSM · Top quark

\section{Introduction}

Since the running LHC provides an opportunity to seek out top quark rare decays, top quark shows great promise in revealing the secret of new physics beyond the standard model (SM). The branching ratios of the flavor changing neutral current (FCNC) of top quark $t \rightarrow c \gamma, t \rightarrow c g, t \rightarrow c h$, $t \rightarrow c Z$ in the SM are highly suppressed [1-8]

$$
\operatorname{Br}(t \rightarrow c \gamma) \sim 5 \times 10^{-14}, \quad \operatorname{Br}(t \rightarrow c g) \sim 5 \times 10^{-12},
$$

\footnotetext{
a e-mail: JLYangJL@ 163.com

be-mail: fengtf@hbu.edu.cn

c e-mail: hbzhang@hbu.edu.cn

de-mail:ninggz@hbu.edu.cn
}

$$
\operatorname{Br}(t \rightarrow c h) \sim 3 \times 10^{-15}, \quad \operatorname{Br}(t \rightarrow c Z) \sim 1 \times 10^{-14},
$$

and beyond the detection capabilities of LHC in the near future. Nevertheless the exotic mechanism from the new physics can enhance those branching ratios drastically [8], which can be detected in the future. The updated upper limits on the branching ratios of LHC are [9-17]

$$
\begin{array}{ll}
\operatorname{Br}(t \rightarrow c \gamma)<1.7 \times 10^{-3}, & \operatorname{Br}(t \rightarrow c g)<2 \times 10^{-4}, \\
\operatorname{Br}(t \rightarrow c h)<2.2 \times 10^{-3}, & \operatorname{Br}(t \rightarrow c Z)<2.3 \times 10^{-4} .
\end{array}
$$

Hence, detecting those rare top quark decays on the LHC provides a good window to search the new physics beyond the SM. Actually several extensions of the SM predict the branching ratios of the rare top decays surpassing the SM evaluations several orders numerically. In Table 1, we present the theoretical predictions on the branching ratios of those rare decays of top quark in some popular new physics extensions of the SM. Those new physics models include the two-Higgs doublet models with flavour-conservation (FC 2HDM) $[2,3,18,19]$ and without flavour-conservation (NFC 2HDM) [20], minimal supersymmetric extension of the SM (MSSM) [21-23], supersymmetry (SUSY) without R-parity $[24,25]$, the Topcolour-assisted Technicolour model (TC2) [26] and the extension with warped extra dimensions (RS) [27]. The results in Refs. [21-23] were obtained when the supersymmetric particles were not constrained strongly by direct searches at the LHC. In addition, the results in Refs. $[21,22]$ under a minimal flavor violating assumption for the soft breaking terms, while Ref. [23] takes into account the off-diagonal terms for the squark matrices. Hence the results in Ref. [23] are larger than those in Refs. [21,22].

In the supersymmetric extensions of the $\mathrm{SM}$, the extension with local $B-L$ gauge symmetry (here employing the abbreviation B-LSSM denoting the extension) draw the attention of physicists, since it provides the candidate for the cold dark matter and the seesaw mechanism to explain the oscillation of neutrinos naturally. The model B-LSSM $[28,29]$ is based on the gauge symmetry group $S U(3)_{C} \otimes S U(2)_{L} \otimes U(1)_{Y} \otimes$ 
Table 1 FCNC decays branching ratios of several SM extension

\begin{tabular}{|c|c|c|c|c|c|c|c|}
\hline Process & $2 \mathrm{HDM}[2,3,18,19]$ & FC 2HDM [20] & MSSM [21,22] & MSSM [23] & $/ R$ SUSY $[24,25]$ & TC2 [26] & RS [27] \\
\hline$t \rightarrow q \gamma$ & $\sim 10^{-6}$ & $\sim 10^{-9}$ & $\sim 10^{-8}$ & $\sim 10^{-6}$ & $\sim 10^{-6}$ & $\sim 10^{-6}$ & $\sim 10^{-9}$ \\
\hline$t \rightarrow q g$ & $\sim 10^{-4}$ & $\sim 10^{-8}$ & $\sim 10^{-6}$ & $\sim 10^{-5}$ & $\sim 10^{-4}$ & $\sim 10^{-4}$ & $\sim 10^{-9}$ \\
\hline$t \rightarrow q h$ & $\sim 10^{-3}$ & $\sim 10^{-5}$ & $\sim 10^{-8}$ & $\sim 10^{-5}$ & $\sim 10^{-6}$ & - & - \\
\hline$t \rightarrow q Z$ & $\sim 10^{-7}$ & $\sim 10^{-10}$ & $\sim 10^{-8}$ & $\sim 10^{-6}$ & $\sim 10^{-7}$ & $\sim 10^{-4}$ & $\sim 10^{-5}$ \\
\hline
\end{tabular}

$U(1)_{B-L}$, where $B$ stands for the baryon number and $L$ stands for the lepton number respectively. Besides accounting elegantly for the existence and smallness of the left-handed neutrino masses, the B-LSSM also alleviates the aforementioned little hierarchy problem of the MSSM [30], because the exotic singlet Higgs and right-handed (s)neutrinos [31-37] release additional parameter space from the LEP, Tevatron and LHC constraints.

In this model, the invariance under $U(1)_{B-L}$ gauge group imposes the the R-parity conservation which is assumed in the MSSM to avoid proton decay. And R-parity conservation can be maintained if $U(1)_{B-L}$ symmetry is broken spontaneously [38]. Furthermore, it could help to understand the origin of R-parity and its possible spontaneous violation in the supersymmetric models [39-41] as well as the mechanism of leptogenesis [42,43]. Moreover, the model can provide much more candidates for the Dark Matter comparing that with the MSSM [44-47].

In this work, we analyze those processes in the B-LSSM, under a minimal flavor violating assumption for the soft breaking terms. In this case, the only source of flavor violation comes from the Cabibbo-Kobayashi-Maskawa (CKM) matrix in the quark sector. And we can explore the effects of new parameters to those processes, with respect to the MSSM. Our presentation is organized as follows. In Sect. 2, the main ingredients of B-LSSM are summarized briefly by introducing the superpotential, the general soft breaking terms and the Higgs sector. In Sect. 3, the branching ratios for $t \rightarrow c \gamma, t \rightarrow c g, t \rightarrow c h$ and $t \rightarrow c Z$ is calculated in the model. The numerical analyses are given in Sect. 4 , and Sect. 5 gives a summary.

\section{The B-LSSM}

In the B-LSSM, one enlarges the local gauge group of the $\mathrm{SM}$ to $S U(3)_{C} \otimes S U(2)_{L} \otimes U(1)_{Y} \otimes U(1)_{B-L}$, where the $U(1)_{B-L}$ is spontaneously broken by the chiral singlet superfields $\hat{\eta}_{1}$ and $\hat{\eta}_{2}$. In literatures there are several popular versions of B-LSSM. Here we adopt the version described in Refs. [48-52] to proceed our analysis, while this version of BLSSM is encoded in SARAH [53-57] which is used to create the mass matrices and interaction vertexes in the B-LSSM. Besides the superfields of the MSSM, the exotic superfields of the B-LSSM are three generations right-handed neutrinos $\hat{v}_{i}^{c} \sim(1,1,0,1)$ and two chiral singlet superfields $\hat{\eta}_{1} \sim(1,1,0,-1), \hat{\eta}_{2} \sim(1,1,0,1)$. Meanwhile, quantum numbers of the matter chiral superfields for quarks and leptons are given by

$$
\begin{aligned}
\hat{Q}_{i} & \sim(3,2,1 / 6,1 / 6), \quad \hat{L}_{i} \sim(1,2,-1 / 2,-1 / 2), \\
\hat{U}_{i} & \sim(3,1,-2 / 3,-1 / 6), \\
\hat{D}_{i} & \sim(3,1,1 / 3,-1 / 6), \quad \hat{E}_{i} \sim(1,1,1,1 / 2)
\end{aligned}
$$

with $i=1,2,3$ denoting the index of generation. In addition, the quantum numbers of two Higgs doublets is assigned as

$$
\begin{aligned}
& \hat{H}_{1}=\left(\begin{array}{l}
H_{1}^{1} \\
H_{1}^{2}
\end{array}\right) \sim(1,2,-1 / 2,0), \\
& \hat{H}_{2}=\left(\begin{array}{l}
H_{2}^{1} \\
H_{2}^{2}
\end{array}\right) \sim(1,2,1 / 2,0) .
\end{aligned}
$$

The corresponding superpotential of the B-LSSM is written as

$$
W=W_{M S S M}+W_{(B-L)} .
$$

Here, $W_{M S S M}$ is the superpotential of the MSSM, and $W_{(B-L)}$ is the sector involving exotic superfields, and

$$
W_{(B-L)}=Y_{\nu, i j} \hat{L}_{i} \hat{H}_{2} \hat{v}_{j}^{c}-\mu^{\prime} \hat{\eta}_{1} \hat{\eta}_{2}+Y_{x, i j} \hat{v}_{i}^{c} \hat{\eta}_{1} \hat{v}_{j}^{c}
$$

where $i, j$ are generation indices. Correspondingly, the soft breaking terms of the B-LSSM are generally given as

$$
\begin{aligned}
\mathcal{L}_{\text {soft }}= & \mathcal{L}_{M S S M}+\left[-M_{B B^{\prime}} \tilde{\lambda}_{B^{\prime}} \tilde{\lambda}_{B}-\frac{1}{2} M_{B^{\prime}} \tilde{\lambda}_{B^{\prime}} \tilde{\lambda}_{B^{\prime}}\right. \\
& -B_{\mu^{\prime}} \tilde{\eta}_{1} \tilde{\eta}_{2}+T_{v}^{i j} H_{2} \tilde{v}_{i}^{c} \tilde{L}_{j}+T_{x}^{i j} \tilde{\eta}_{1} \tilde{v}_{i}^{c} \tilde{v}_{j}^{c} \\
& + \text { h.c. }]-m_{\tilde{\eta}_{1}}^{2}\left|\tilde{\eta}_{1}\right|^{2}-m_{\tilde{\eta}_{2}}^{2}\left|\tilde{\eta}_{2}\right|^{2}-m_{\tilde{v}, i j}^{2}\left(\tilde{v}_{i}^{c}\right)^{*} \tilde{v}_{j}^{c},
\end{aligned}
$$

with $\lambda_{B}, \lambda_{B^{\prime}}$ denoting the gaugino of $U(1)_{Y}$ and $U(1)_{(B-L)}$ respectively, $\mathcal{L}_{M S S M}$ is the soft breaking terms in MSSM. The local gauge symmetry $S U(2)_{L} \otimes U(1)_{Y} \otimes U(1)_{B-L}$ breaks down to the electromagnetic symmetry $U(1)_{e m}$ as the Higgs fields receive vacuum expectation values (VEVs):

$H_{1}^{1}=\frac{1}{\sqrt{2}}\left(v_{1}+\operatorname{Re} H_{1}^{1}+i \operatorname{Im} H_{1}^{1}\right)$, 
$H_{2}^{2}=\frac{1}{\sqrt{2}}\left(v_{2}+\operatorname{Re} H_{2}^{2}+i \operatorname{Im} H_{2}^{2}\right)$,

$\tilde{\eta}_{1}=\frac{1}{\sqrt{2}}\left(u_{1}+\operatorname{Re} \tilde{\eta}_{1}+i \operatorname{Im} \tilde{\eta}_{1}\right)$,

$\tilde{\eta}_{2}=\frac{1}{\sqrt{2}}\left(u_{2}+i \operatorname{Re} \tilde{\eta}_{2}+i \operatorname{Im} \tilde{\eta}_{2}\right)$.

For convenience, we define $u^{2}=u_{1}^{2}+u_{2}^{2}, v^{2}=v_{1}^{2}+v_{2}^{2}$ and $\tan \beta^{\prime}=\frac{u_{2}}{u_{1}}$ in analogy to the ratio of the MSSM VEVs $\left(\tan \beta=\frac{v_{2}}{v_{1}}\right)$.

The presence of two Abelian groups gives rise to a new effect absent in the MSSM or other SUSY models with just one Abelian gauge group: the gauge kinetic mixing. It results from the invariance principle allows the Lagrangian to include a mixing term between the strength tensors of gauge fields associated with the $U(1)$ gauge groups, $-\kappa_{Y, B L} A_{\mu}^{\prime Y} A^{\prime \mu, B L}$, where $A_{\mu}^{\prime Y}, A^{\prime \mu, B L}$ denote the gauge fields associated with the two $U(1)$ gauge groups, $Y, B-L$ corresponding to the hypercharge and $\mathrm{B}-\mathrm{L}$ charge respectively, $\kappa_{Y, B L}$ is an antisymmetric tensor which includes the mixing of $U(1)_{Y}$ and $U(1)_{B-L}$ gauge fields. This mixing couples the B-L sector to the MSSM sector, and even if it is set to zero at $M_{G U T}$, it can be induced through RGEs [58-64]. In practice, it turns out that it is easier to work with non-canonical covariant derivatives instead of off-diagonal field-strength tensors. However, both approaches are equivalent [65]. Hence in the following, we consider covariant derivatives of the form

$$
D_{\mu}=\partial_{\mu}-i(Y, B-L)\left(\begin{array}{cc}
g_{Y}, & g_{Y B}^{\prime} \\
g_{B Y}^{\prime}, & g_{B-L}
\end{array}\right)\left(\begin{array}{c}
A_{\mu}^{\prime Y} \\
A_{\mu}^{\prime B L}
\end{array}\right) .
$$

As long as the two Abelian gauge groups are unbroken, we still have the freedom to perform a change of the basis

$$
D_{\mu}=\partial_{\mu}-i(Y, B-L)\left(\begin{array}{cc}
g_{Y}, & g_{Y B}^{\prime} \\
g_{B Y}^{\prime} & g_{B-L}^{\prime}
\end{array}\right) R^{T} R\left(\begin{array}{c}
A_{\mu}^{\prime Y} \\
A_{\mu}^{\prime B L}
\end{array}\right),
$$

where $R$ is a $2 \times 2$ orthogonal matrix. Choosing $R$ in a proper form, one can write the coupling matrix as

$$
\left(\begin{array}{cc}
g_{Y}, & g_{Y B}^{\prime} \\
g_{B Y}^{\prime}, & g_{B-L}
\end{array}\right) R^{T}=\left(\begin{array}{cc}
g_{1}, & g_{Y B} \\
0, & g_{B}
\end{array}\right),
$$

where $g_{1}$ corresponds to the measured hypercharge coupling which is modified in B-LSSM as given along with $g_{B}$ and $g_{Y B}$ in $[66,67]$. Then, we can redefine the $U(1)$ gauge fields

$$
R\left(\begin{array}{c}
A_{\mu}^{\prime Y} \\
A_{\mu}^{\prime B L}
\end{array}\right)=\left(\begin{array}{c}
A_{\mu}^{Y} \\
A_{\mu}^{B L}
\end{array}\right) .
$$

Immediate interesting consequence of the gauge kinetic mixing arise in various sectors of the model as discused in the subsequent analysis. Firstly, $A^{B L}$ boson mixes at the tree level with the $A^{Y}$ and $V^{3}$ bosons. In the basis $\left(A^{Y}, V^{3}, A^{B L}\right)$, the correponding mass matrix reads,

$$
\left(\begin{array}{ccc}
\frac{1}{8} g_{1}^{2} v^{2} & -\frac{1}{8} g_{1} g_{2} v^{2} & \frac{1}{8} g_{1} g_{Y B} v^{2} \\
-\frac{1}{8} g_{1} g_{2} v^{2} & \frac{1}{8} g_{2}^{2} v^{2} & -\frac{1}{8} g_{2} g_{Y B} v^{2} \\
\frac{1}{8} g_{1} g_{Y B} v^{2} & -\frac{1}{8} g_{2} g_{Y B} v^{2} & \frac{1}{8} g_{Y B}^{2} v^{2}+\frac{1}{8} g_{B}^{2} u^{2}
\end{array}\right) .
$$

This mass matrix can be diagonalized by a unitary mixing matrix, which can be expressed by two mixing angles $\theta_{W}$ and $\theta_{W}^{\prime}$ as

$$
\left(\begin{array}{c}
\gamma \\
Z \\
Z^{\prime}
\end{array}\right)=\left(\begin{array}{ccc}
\cos \theta_{W} & \sin \theta_{W} & 0 \\
-\sin \theta_{W} \cos \theta_{W}^{\prime} & \cos \theta_{W} \cos \theta_{W}^{\prime} & \sin \theta_{W}^{\prime} \\
\sin \theta_{W} \sin \theta_{W}^{\prime} & -\cos \theta_{W}^{\prime} \sin \theta_{W}^{\prime} & \cos \theta_{W}^{\prime}
\end{array}\right)\left(\begin{array}{c}
A^{Y} \\
V^{3} \\
A^{B L}
\end{array}\right) .
$$

Then $\sin ^{2} \theta_{W}^{\prime}$ can be written as

$$
\begin{aligned}
& \sin ^{2} \theta_{W}^{\prime}=\frac{1}{2} \\
& -\frac{\left(g_{Y B}^{2}-g_{1}^{2}-g_{2}^{2}\right) x^{2}+4 g_{B}^{2}}{2 \sqrt{\left(g_{Y B}^{2}+g_{1}^{2}+g_{2}^{2}\right) x^{4}+8 g_{B}^{2}\left(g_{Y B}^{2}-g_{1}^{2}-g_{2}^{2} x^{2}\right)+16 g_{B}^{2}}},
\end{aligned}
$$

where $x=\frac{v}{u}$. Compared with the MSSM, this $Z-Z^{\prime}$ mixing makes new contributions to the $t \rightarrow c Z$ decay channel, and the order of magnitude of $\sin \theta_{W}^{\prime}$ is about $\mathcal{O}\left(10^{-2}\right)[68,69]$. The exact eigenvalues of Eq.(13) are given by

$$
\begin{aligned}
m_{\gamma}^{2}= & 0, \\
m_{Z, Z^{\prime}}^{2}= & \frac{1}{8}\left(\left(g_{1}^{2}+g_{2}^{2}+g_{Y B}^{2}\right) v^{2}+4 g_{B}^{2} u^{2}\right. \\
& \left.\mp \sqrt{\left(g_{1}^{2}+g_{2}^{2}+g_{Y B}^{2}\right)^{2} v^{4}+8\left(g_{Y B}^{2}-g_{1}^{2}-g_{2}^{2}\right) g_{B}^{2} v^{2} u^{2}+16 g_{B}^{4} u^{4}}\right),
\end{aligned}
$$

In addition, the charged Higgs boson and $W$ gauge boson mass can be written as

$$
\begin{aligned}
m_{H^{ \pm}}^{2} & =\frac{4 B_{\mu}\left(1+\tan \beta^{2}\right)+g_{2}^{2} v_{1}^{2} \tan \beta\left(1+\tan \beta^{2}\right)}{4 \tan \beta}, \\
m_{W}^{2} & =\frac{1}{4} g_{2}^{2} v^{2} .
\end{aligned}
$$

Then the gauge kinetic mixing leads to the mixing between the $H_{1}^{1}, H_{2}^{2}, \tilde{\eta_{1}}, \tilde{\eta_{2}}$ at the tree level. In the basis $\left(\operatorname{Re} H_{1}^{1}\right.$, $\left.\operatorname{Re} H_{2}^{2}, \operatorname{Re} \tilde{\eta}_{1}, \operatorname{Re} \tilde{\eta}_{2}\right)$, the tree level mass squared matrix for scalar Higgs bosons is given by 


$$
\begin{aligned}
& M_{h}^{2}= u^{2} \\
& \times\left(\begin{array}{cccc}
\frac{1}{4} \frac{g^{2} x^{2}}{1+\tan \beta^{2}}+n^{2} \tan \beta-\frac{1}{4} g^{2} \frac{x^{2} \tan \beta}{1+\tan ^{2} \beta}-n^{2} & \frac{1}{2} g_{B} g_{Y B} \frac{x}{T} & -\frac{1}{2} g_{B} g_{Y B} \frac{x \tan \beta^{\prime}}{T} \\
-\frac{1}{4} g^{2} \frac{x^{2} \tan \beta}{1+\tan ^{2} \beta}-n^{2} & \frac{1}{4} \frac{g^{2} \tan ^{2} \beta x^{2}}{1+\tan \beta^{2}}+\frac{n^{2}}{\tan \beta} & \frac{1}{2} g_{B} g_{Y B} \frac{x \tan \beta}{T} & \frac{1}{2} g_{B} g_{Y B} \frac{x \tan \beta \tan \beta^{\prime}}{T} \\
\frac{1}{2} g_{B} g_{Y B} \frac{x}{T} & \frac{1}{2} g_{B} g_{Y B} \frac{x \tan \beta}{T} & \frac{g_{B}^{2}}{1+\tan ^{2} \beta^{\prime}}+\tan \beta^{\prime} N^{2}-g_{B}^{2} \frac{\tan \beta^{\prime}}{1+\tan ^{2} \beta^{\prime}}-N^{2} \\
-\frac{1}{2} g_{B} g_{Y B} \frac{x \tan \beta^{\prime}}{T} & \frac{1}{2} g_{B} g_{Y B} \frac{x \tan \beta \tan \beta^{\prime}}{T} & -g_{B}^{2} \frac{\tan \beta^{\prime}}{1+\tan ^{2} \beta^{\prime}}-N^{2} & g_{B}^{2} \frac{\tan ^{2} \beta^{\prime}}{1+\tan ^{2} \beta^{\prime}}+\frac{N^{2}}{\tan \beta^{\prime}}
\end{array}\right)
\end{aligned}
$$

where $g^{2}=g_{1}^{2}+g_{2}^{2}+g_{Y B}^{2}, T=\sqrt{1+\tan ^{2} \beta} \sqrt{1+\tan ^{2} \beta^{\prime}}$, $n^{2}=\frac{\operatorname{Re} B \mu}{u^{2}}$ and $N^{2}=\frac{\operatorname{Re} B \mu^{\prime}}{u^{2}}$, respectively. Compared the MSSM, this new mixing in the B-LSSM can affect the theoretical prediction of the process $t \rightarrow c h$.

Including the leading-log radiative corrections from stop and top quark, the mass of the SM-like Higgs boson can be written as [70-72]

$$
\begin{aligned}
\Delta m_{h}^{2}= & \frac{3 m_{t}^{4}}{2 \pi v^{2}}\left[\left(\tilde{t}+\frac{1}{2}+\tilde{X}_{t}\right)\right. \\
& \left.+\frac{1}{16 \pi^{2}}\left(\frac{3 m_{t}^{2}}{2 v^{2}}-32 \pi \alpha_{3}\right)\left(\tilde{t}^{2}+\tilde{X}_{t} \tilde{t}\right)\right], \\
\tilde{t}= & \log \frac{M_{S}^{2}}{m_{t}^{2}}, \quad \tilde{X}_{t}=\frac{2 \tilde{A}_{t}^{2}}{M_{S}^{2}}\left(1-\frac{\tilde{A}_{t}^{2}}{12 M_{S}^{2}}\right),
\end{aligned}
$$

where $\alpha_{3}$ is the strong coupling constant, $M_{S}=\sqrt{m_{\tilde{t}_{1}} m_{\tilde{t}_{2}}}$ with $m_{\tilde{t}_{1,2}}$ denoteting the stop masses, $\tilde{A}_{t}=A_{t}-\mu \cot \beta$ with $A_{t}=T_{u, 33}$ being the trilinear Higgs stop coupling and $\mu$ denoting the Higgsino mass parameter. Then the SM-like Higgs mass can be written as

$$
m_{h}=\sqrt{\left(m_{h_{1}}^{0}\right)^{2}+\Delta m_{h}^{2}},
$$

where $m_{h_{1}}^{0}$ denotes the lightest tree-level Higgs mass.

Meanwhile, additional D-terms contribute to the mass matrices of the squarks and sleptons, and down type squarks affect the subsequent analysis. On the basis $\left(\tilde{d}_{L}, \tilde{d}_{R}\right)$, mass matrix for down type squarks is given by

$$
\begin{aligned}
m_{\tilde{d}}^{2}= & \left(\begin{array}{cc}
m_{d L}, & \frac{1}{\sqrt{2}}\left(v_{1} T_{d}^{\dagger}-v_{2} \mu Y_{d}^{\dagger}\right) \\
\frac{1}{\sqrt{2}}\left(v_{1} T_{d}-v_{2} \mu^{*} Y_{d}\right), & m_{d R}
\end{array}\right), \\
m_{d L}= & \frac{1}{24}\left(2 g_{B}\left(g_{B}+g_{Y B}\right)\left(u_{2}^{2}-u_{1}^{2}\right)\right. \\
& \left.+\left(3 g_{2}^{2}+g_{1}^{2}+g_{Y B}^{2}+g_{B} g_{Y B}\right)\left(v_{2}^{2}-v_{1}^{2}\right)\right) \\
& +m_{\tilde{q}}^{2}+\frac{v_{1}^{2}}{2} Y_{d}^{\dagger} Y_{d}, \\
m_{d R}= & \frac{1}{24}\left(2 g_{B}\left(g_{B}-2 g_{Y B}\right)\left(u_{1}^{2}-u_{2}^{2}\right)+2\left(g_{1}^{2}\right.\right. \\
& \left.\left.+g_{Y B}^{2}-\frac{1}{2} g_{B} g_{Y B}\right)\left(v_{2}^{2}-v_{1}^{2}\right)\right) \\
& +m_{\tilde{d}}^{2}+\frac{v_{1}^{2}}{2} Y_{d}^{\dagger} Y_{d} .
\end{aligned}
$$

It can be noted that new gauge coupling constants $g_{B}$ and $g_{Y B}$, with respect to the MSSM, affect the masses of down type squarks significantly when $u$ is large.

\section{Theoretical calculation on $t \rightarrow c \gamma, c g, c Z$ and $c h$ processes}

In this section, we analyze one-loop radiative corrections to the rare decay processes of top quark $t \rightarrow c \gamma, c g, c Z$ and $c h$ in the B-LSSM. Here the contributions from self-energy are attributed to the renormalization of external quarks wave functions. The dominating triangle diagrams contributing to the rare top quark decay processes $t \rightarrow c \gamma, c g, c Z, c h$ are presented in Figs. 1, 2, respectively.

In the B-LSSM, the corresponding amplitude for the rare decay process $t \rightarrow c \gamma, g, Z$ is written as

$$
\begin{aligned}
\mathcal{M}_{t c V}= & (4 \pi)^{-2} \varepsilon^{\mu} \bar{u}_{c}\left(p^{\prime}\right)\left(\kappa_{V L 1} \gamma_{\mu} P_{L}+i \kappa_{V L 2} \sigma_{\mu \nu} q^{v} P_{L}\right. \\
& +(L \rightarrow R)) u_{t}(p),
\end{aligned}
$$

where $V$ denotes $\gamma, g, Z, u_{t}$ and $u_{c}$ denote the wave functions of top quark and charm quark, $p$ is the momentum of top quark, $p^{\prime}$ is the momentum of charm quark, $q$ is the momentum of vector boson, and $\varepsilon^{\mu}$ denotes the polarization vector of photon, gluon and $\mathrm{Z}$ boson. Correspondingly, the coefficients $\kappa_{V L i}$ and $\kappa_{V R i}(i=1,2)$ are originating from those Feynman diagrams in Fig. 1. The picture shows that, compared the MSSM, the new definition of the down type squark mass matrix and the corresponding rotation matrix affect the predictions on the processes $t \rightarrow c \gamma, c g, c Z$ in the B-LSSM. In addition, the $Z-Z^{\prime}$ mixing in Eq. (13) also makes new contributions to the $t \rightarrow c Z$ decay channel.

Then in order to explain how the calculation of the feynman diagrams in Fig. 1 has been performed, we will take the calculation of Fig. 1(1) below for example. The corresponding amplitude can be written as

$$
\begin{aligned}
\mathcal{M}_{t c V}^{(1)}= & \varepsilon^{\mu} \bar{u}_{c}\left(p^{\prime}\right) \mu^{4-D} \int \frac{d^{D} k}{(2 \pi)^{D}}\left(i A_{\bar{c}} \tilde{D}_{k} \tilde{\chi}_{j}^{+}{ }_{L} P_{L}\right. \\
& \left.+i A_{\bar{c} \tilde{D}_{k} \tilde{\chi}_{j}^{+} R} P_{R}\right) \frac{i}{\not p-\not k-m_{\tilde{\chi}_{j}^{+}}}\left(i A_{\tilde{\chi}_{j}^{+} \tilde{D}_{i} t L} P_{L}\right.
\end{aligned}
$$




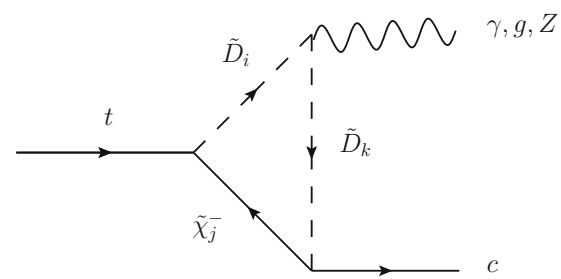

(1)

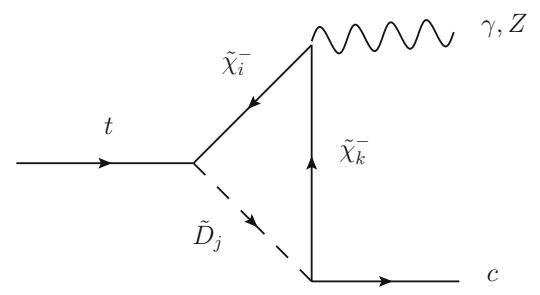

(4)

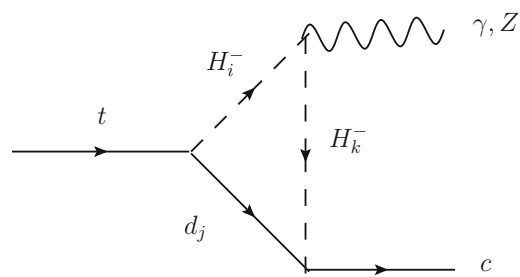

(2)

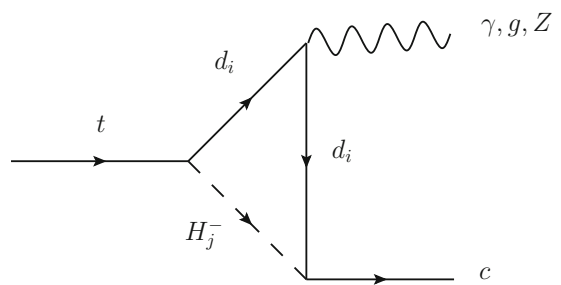

(5)

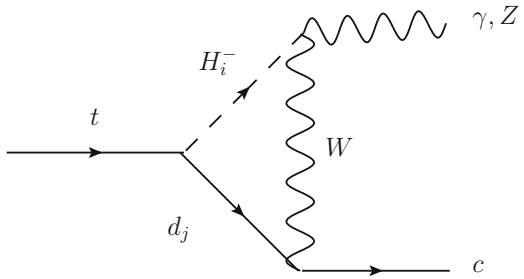

(3)

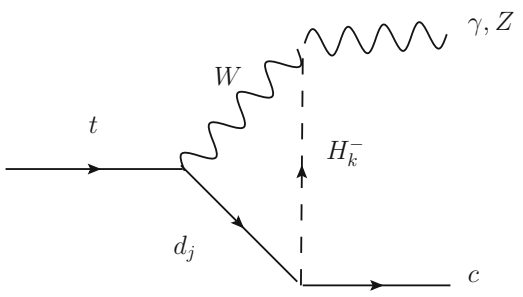

(6)

Fig. 1 The dominating Feynman diagrams contributing to $t \rightarrow c \gamma, g, Z$ in the B-LSSM

$$
\begin{aligned}
& \left.+i A_{\tilde{\chi}_{j}^{+}+\tilde{D}_{i} t R} P_{R}\right) \frac{i}{k^{2}-m_{\tilde{D}_{i}}^{2}}\left(i B_{V \tilde{D}_{i} \tilde{D}_{k}}\left(-2 k_{\mu}+q_{\mu}\right)\right) \\
& \times \frac{i}{\left(k-q^{2}\right)-m_{\tilde{D}_{k}}^{2}} u_{t}(p),
\end{aligned}
$$

where $A_{\bar{c} \tilde{D}_{k} \tilde{\chi}_{j}^{+} L, R}, A_{\tilde{\chi}_{j}^{+} \tilde{D}_{i} t L, R}, B_{V \tilde{D}_{i} \tilde{D}_{k}}$ denote the constant parts of the interaction vertex about $\bar{c} \tilde{D}_{k} \tilde{\chi}_{j}^{+}, \overline{\tilde{\chi}}_{j}^{+} \tilde{D}_{i} t, V \tilde{D}_{i}$ $\tilde{D}_{k}$ respectively, $L$ and $R$ in subscript denote the left-hand part and right-hand part, and all of them can be got through SARAH. Apply the transverse wave condition and Dirac equation, the amplitude can be simplify as

$$
\begin{aligned}
\mathcal{M}_{t c V}^{(1)}= & \frac{1}{16 \pi^{2}} \varepsilon^{\mu} \bar{u}_{c}\left(p^{\prime}\right)\left(\kappa_{V L 1}^{(1)} \gamma_{\mu} P_{L}+i \kappa_{V L 2}^{(1)} \sigma_{\mu \nu} q^{\nu} P_{L}\right. \\
& +(L \rightarrow R)) u_{t}(p)
\end{aligned}
$$

where

$$
\begin{aligned}
& \kappa_{V L 1}^{(1)}=-i B_{V \tilde{D}_{i} \tilde{D}_{k}}\left[\left(m_{t} m_{c}\left(-C_{2}-C_{22}\right)-2 C_{00}\right)\right. \\
& \times A_{\overline{\tilde{\chi}}_{j}^{+} \tilde{D}_{i} t R} A_{\bar{c} \tilde{D}_{k} \tilde{\chi}_{j}^{+} L}+\left(m _ { t } ^ { 2 } \left(-C_{2}-C_{22}\right.\right. \\
& \left.\left.-C_{12}\right)+m_{c}^{2} C_{12}\right) A_{\bar{\chi}_{j}^{+} \tilde{D}_{i} t L} A_{\bar{c} \tilde{D}_{k} \tilde{\chi}_{j}^{+} R} \\
& -m_{\tilde{\chi}_{j}^{+}} m_{c} C_{2} A_{\tilde{\chi}_{j}^{+} \tilde{D}_{i} t L} A_{\bar{c} \tilde{D}_{k} \tilde{\chi}_{j}^{+} L} \\
& \left.-m_{\tilde{\chi}_{j}^{+}} m_{t} C_{2} A_{\bar{\chi}_{j}^{+} \tilde{D}_{i} t R} A_{\bar{c} \tilde{D}_{k} \tilde{\chi}_{j}^{+} R}\right] \text {, } \\
& \kappa_{V L 2}^{(1)}=-i B_{V \tilde{D}_{i} \tilde{D}_{k}}\left[m_{t}\left(-C_{2}-C_{22}-C_{12}\right)\right. \\
& \times A_{\tilde{\chi}_{j}^{+}} \tilde{D}_{i} t R A_{\bar{c} \tilde{D}_{k} \tilde{\chi}_{j}^{+} L} \\
& -m_{\tilde{\chi}_{j}^{+}} C_{2} A_{\tilde{\chi}_{j}^{+} \tilde{D}_{i} t L} A_{\bar{c} \tilde{D}_{k} \tilde{\chi}_{j}^{+} L} \\
& \left.+m_{c} C_{12} A_{t \tilde{D}_{i} \tilde{\chi}_{j}^{+} L} A_{c \tilde{D}_{k} \tilde{\chi}_{j}^{+} R}\right] \text {, }
\end{aligned}
$$

$\kappa_{V R 1,2}^{(1)}=\kappa_{V L 1,2}^{(1)}(L \leftrightarrow R)$.

Here $C_{0}, C_{1}, C_{2}, C_{00}, C_{11}, C_{12}, C_{22}$ are Passarino-Veltman scalar functions [73], and the argument above is $\left[m_{V}^{2}, m_{c}^{2}, m_{t}^{2}\right.$, $\left.m_{\tilde{D}_{i}}^{2}, m_{\tilde{D}_{k}}^{2}, m_{\tilde{\chi}_{j}^{+}}^{2}\right]$. The other diagrams corresponding to $t \rightarrow$ $c V$ can be calculated similarly and contribute to the operators $\gamma_{\mu} P_{L, R}$ and $i \sigma_{\mu \nu} q^{\nu} P_{L, R}$.

In the effective coupling for $h \bar{c} t$, there are only two effective operators:

$$
\mathcal{M}_{t c h}=(4 \pi)^{-2} \bar{u}_{c}\left(p^{\prime}\right)\left(\kappa_{h L} P_{L}+\kappa_{h R} P_{R}\right) u_{t}(p),
$$

the coefficients $\kappa_{h L}, \kappa_{h R}$ are originating from those Feynman diagrams in Fig. 2:

$$
\begin{aligned}
& \kappa_{h L}=\sum_{a=1}^{6} \kappa_{h L}^{(a)}, \\
& \kappa_{h R}=\sum_{a=1}^{6} \kappa_{h R}^{(a)},
\end{aligned}
$$

where the contributions $\kappa_{h L}^{(a)}, \kappa_{h R}^{(a)}$ depend on the relevant Feynman diagrams in the model. Figure 2 shows that, except for the new contributions from down type squarks, the mixing between the Higgs doublets and the exotic singlets $\tilde{\eta}_{1,2}$ also affects the $t \rightarrow c h$ decay channel. And we will take the calculation of Fig. 2(1) below for example. The amplitude can be given as

$$
\begin{aligned}
\mathcal{M}_{t c h}^{(1)}= & \bar{u}_{c}\left(p^{\prime}\right) \mu^{4-D} \int \frac{d^{D} k}{(2 \pi)^{D}}\left(i A_{\bar{c} \tilde{D}_{k} \tilde{\chi}_{j}^{+} L} P_{L}\right. \\
& \left.+i A_{\bar{c} \tilde{D}_{k} \tilde{\chi}_{j}^{+} R} P_{R}\right) \frac{i}{\not p-\not k-m_{\tilde{\chi}_{j}^{+}}}\left(i A_{\tilde{\chi}_{j}^{+}} \tilde{D}_{i} t L\right.
\end{aligned}
$$




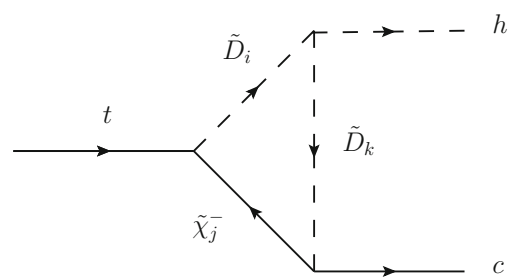

(1)

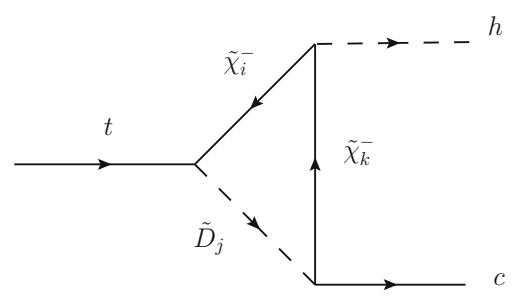

(4)

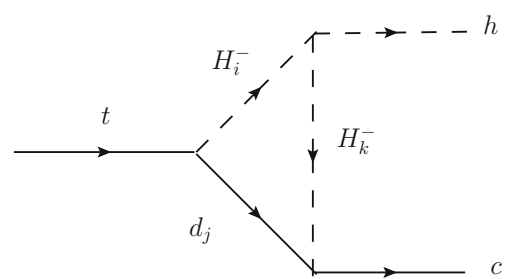

(2)

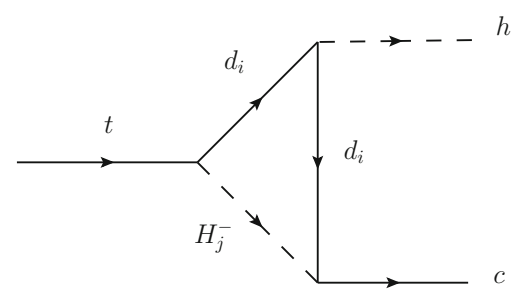

(5)

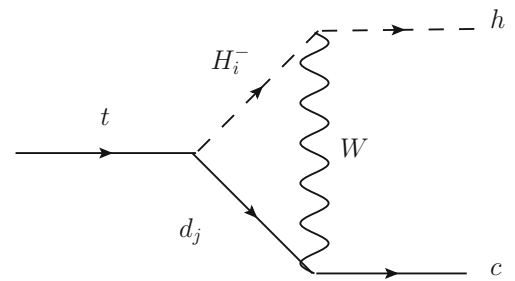

(3)

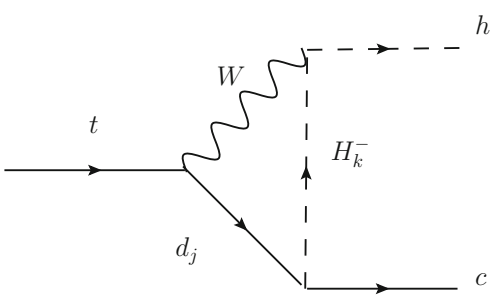

(6)

Fig. 2 The Feynman diagrams contributing to $t \rightarrow c h$ in the B-LSSM

$$
\begin{aligned}
& \left.+i A_{\overline{\tilde{\chi}}_{j}^{+} \tilde{D}_{i} t R} P_{R}\right) \frac{i}{k^{2}-m_{\tilde{D}_{i}}^{2}}\left(i B_{h \tilde{D}_{i} \tilde{D}_{k}}\right) \\
& \times \frac{i}{\left(k-q^{2}\right)-m_{\tilde{D}_{k}}^{2}} u_{t}(p),
\end{aligned}
$$

Apply the Dirac equation, the amplitude can be simplify as

$$
\mathcal{M}_{t c h}^{(1)}=\frac{1}{16 \pi^{2}} \bar{u}_{c}\left(p^{\prime}\right)\left(\kappa_{h L} P_{L}+\kappa_{h R} P_{R}\right) u_{t}(p)
$$

where

$$
\begin{aligned}
\kappa_{h L}^{(1)}= & -i B_{h \tilde{D}_{i} \tilde{D}_{k}}\left[m_{t}\left(C_{0}+C_{1}+C_{2}\right) A_{\bar{f} \tilde{D}_{i} t R} A_{\bar{c} \tilde{D}_{k} \tilde{\chi}_{j}^{+} L}\right. \\
& -m_{c} C_{1} A_{\tilde{\chi}_{j}^{+} \tilde{D}_{i} t L} A_{\bar{c} \tilde{D}_{k} \tilde{\chi}_{j}^{+} R} \\
& +m_{\tilde{\chi}_{j}^{+}} C_{0} A_{\overline{\tilde{\chi}}_{j}^{+} \tilde{D}_{i} t L} A_{\left.\bar{c} \tilde{D}_{k} \tilde{\chi}_{j}^{+} L\right],} \\
\kappa_{h R}^{(1)}= & \kappa_{h L}^{(1)}(L \leftrightarrow R) .
\end{aligned}
$$

the argument in Passarino-Veltman scalar functions above is $\left[m_{h}^{2}, m_{c}^{2}, m_{t}^{2}, m_{\tilde{D}_{i}}^{2}, m_{\tilde{D}_{k}}^{2}, m_{\tilde{\chi}_{j}^{+}}^{2}\right]$. The other diagrams corresponding to $t \rightarrow c h$ can be calculated similarly and contribute to the operators $P_{L}$ and $P_{R}$.

Based on Eqs. (23) and (29), the corresponding branching ratios of the rare decay processes of top quark respectively read as

$$
\begin{aligned}
& \operatorname{Br}(t \rightarrow c V) \\
& \quad=\frac{\left|\mathcal{M}_{t c V}\right|^{2} \sqrt{\left(\left(m_{t}+m_{V}\right)^{2}-m_{c}^{2}\right)\left(\left(m_{t}-m_{V}\right)^{2}-m_{c}^{2}\right)}}{32 \pi m_{t}^{3} \Gamma_{\text {total }}}, \\
& \operatorname{Br}(t \rightarrow c h) \\
& \quad=\frac{\left|\mathcal{M}_{t c h}\right|^{2} \sqrt{\left(\left(m_{t}+m_{h}\right)^{2}-m_{c}^{2}\right)\left(\left(m_{t}-m_{h}\right)^{2}-m_{c}^{2}\right)}}{32 \pi m_{t}^{3} \Gamma_{\text {total }}} .
\end{aligned}
$$

where $\Gamma_{\text {total }}=1.4 \mathrm{GeV}[74]$ is the total decay width of top quark.

\section{Numerical analyses}

In this section, we present the numerical results of $\mathrm{Br}(t \rightarrow$ $c \gamma, c g, c Z, c h)$ with the help of LoopTools and FeynCalc $[75,76]$. The relevant SM input parameters are chosen as $m_{W}=80.385 \mathrm{GeV}, m_{Z}=90.19 \mathrm{GeV}, \alpha_{e m}\left(m_{Z}\right)=$ $1 / 128.9, \quad \alpha_{s}\left(m_{Z}\right)=0.118, \quad m_{t}=173.5 \mathrm{GeV}, \quad m_{c}=$ $1.275 \mathrm{GeV}$. Meanwhile the CKM matrix is [74]

$$
\left(\begin{array}{lll}
0.97417 & 0.2248 & 4.09 \times 10^{-3} \\
-0.22 & 0.995 & 4.05 \times 10^{-2} \\
8.2 \times 10^{-3} & -4 \times 10^{-2} & 1.009
\end{array}\right)
$$

The updated experimental data [77] on searching $Z^{\prime}$ indicates $M_{Z^{\prime}} \geq 4.05 \mathrm{TeV}$ at $95 \%$ Confidence Level (CL), and Refs. [78,79] give us an upper bound on the ratio between the $Z^{\prime}$ mass and its gauge coupling at $99 \% \mathrm{CL}$ as

$$
M_{Z^{\prime}} / g_{B} \geq 6 \mathrm{TeV} \text {. }
$$

In order to coincide with the experimental data, we choose $M_{Z^{\prime}}=4.2 \mathrm{TeV}$ in our numerical analysis, then the scope of $g_{B}$ is limited to $0<g_{B} \leq 0.7$. The LHC experimental data also constrain $\tan \beta^{\prime}<1.5$. Considering the constraints from the experiments [80], for those parameters in Higgsino and gaugino sectors, we appropriately fix $M_{1}=500 \mathrm{GeV}, M_{2}=600 \mathrm{GeV}, M_{B B^{\prime}}=$ $500 \mathrm{GeV}, \quad M_{B L}=600 \mathrm{GeV}, \mu=700 \mathrm{GeV}, \quad \mu^{\prime}=$ $800 \mathrm{GeV}$. For simplify, we set $m_{H^{ \pm}}=2 \mathrm{TeV}, B_{\mu}^{\prime}=$ $5 \times 10^{5} \mathrm{GeV}^{2}, \quad T_{u}=T_{d}=\operatorname{diag}\left(1,1, A_{t}\right) \mathrm{TeV}$. In addi- 


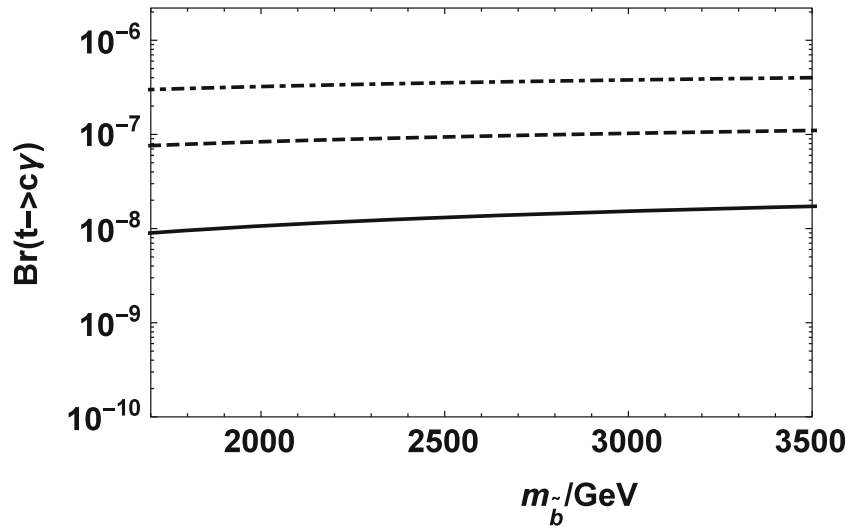

(a)

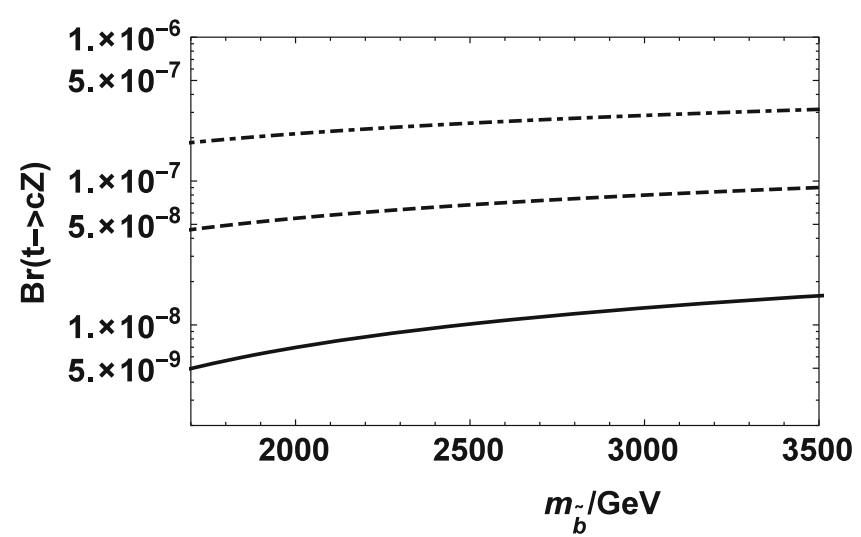

(c)

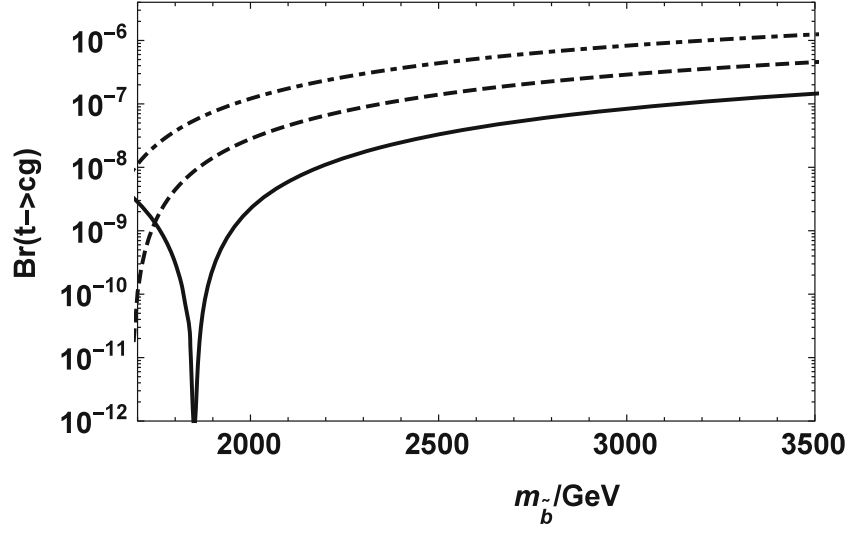

(b)

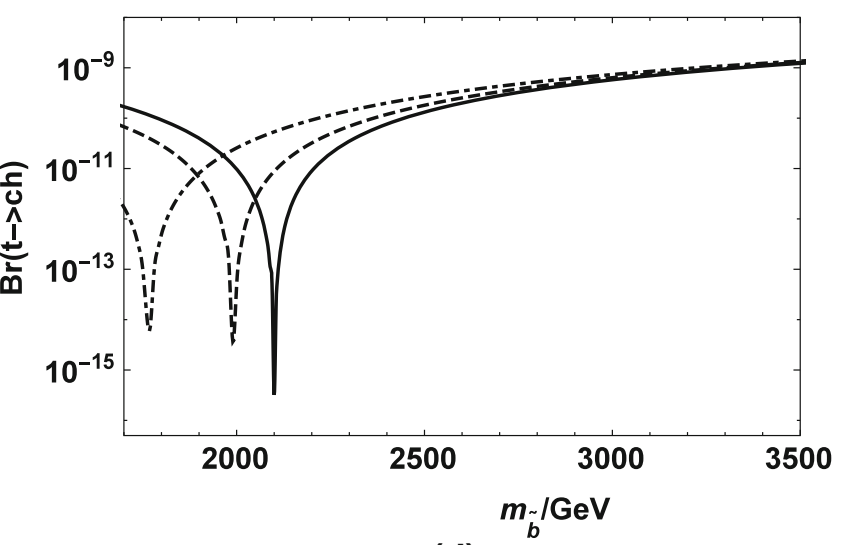

(d)

Fig. $3 \operatorname{Br}(t \rightarrow c \gamma)(\mathbf{a}), \operatorname{Br}(t \rightarrow c g)(\mathbf{b}), \operatorname{Br}(t \rightarrow c Z)(\mathbf{c}), \operatorname{Br}(t \rightarrow c h)(\mathbf{d})$ versus $m_{\tilde{b}}$ for $\tan \beta=15$ (solid line), $\tan \beta=$ 25 (dashed line), $\tan \beta=35$ (dot-dashed line) are plotted

tion, the first two generations of squarks are strongly constrained by direct searches at the LHC $[81,82]$ and the third generation squark masses are not constrained by the LHC as strong as the first two generations. Therefore we take $m_{\tilde{q}}^{2}=m_{\tilde{d}}^{2}=m_{\tilde{u}}^{2}=\operatorname{diag}\left(2 \mathrm{TeV}, 2 \mathrm{TeV}, m_{\tilde{b}}\right)$, and the discussion about the observed Higgs singal in Refs. [34,83] limits $m_{\tilde{b}} \gtrsim 1.5 \mathrm{TeV}$.

It's well known that the experimental observation on $\operatorname{Br}\left(\bar{B} \rightarrow X_{S} \gamma\right)$ limits the relevant parameters strongly, hence we consider the constraint from $\bar{B} \rightarrow X_{s} \gamma$ in this work. In addition, we further consider the constraint from $B_{s}^{0} \rightarrow \mu^{+} \mu^{-}$, which might also limit our numerical analyses [84]. The latest experimental data for $\operatorname{Br}\left(\bar{B} \rightarrow X_{S} \gamma\right)$ and $\operatorname{Br}\left(B_{s}^{0} \rightarrow \mu^{+} \mu^{-}\right)$read [74]

$\operatorname{Br}\left(\bar{B} \rightarrow X_{S} \gamma\right)=(3.49 \pm 0.19) \times 10^{-4}$,

$\operatorname{Br}\left(B_{s}^{0} \rightarrow \mu^{+} \mu^{-}\right)=\left(2.9_{-0.6}^{+0.7}\right) \times 10^{-9}$.

We also need to consider the constraint of SM-like Higgs boson mass [84]. Taking $\tan \beta^{\prime}=1.1, g_{B}=0.2, g_{Y B}=$ $-0.6, A_{t}=-1.5$ and considering the restrictions from
B physics and concrete Higgs boson mass, then letting $m_{\tilde{b}}$ runs from $1.5 \mathrm{TeV}$ to $4 \mathrm{TeV}$ and $\tan \beta$ runs from 2 to 40 , the allowed region of them are

$$
10<\tan \beta<40, \quad 1700 \mathrm{GeV}<m_{\tilde{b}}<3500 \mathrm{GeV} .
$$

Then we plot $\mathrm{Br}(t \rightarrow c \gamma), \operatorname{Br}(t \rightarrow c g), \operatorname{Br}(t \rightarrow c Z)$ and $\operatorname{Br}(t \rightarrow c h)$ versus $m_{\tilde{b}}$ in Fig. 3a-d, where the solid line, dashed line, dot-dashed line denote $\tan \beta=15,25,35$ respectively. From the picture, we can see that $\operatorname{Br}(t \rightarrow$ $c \gamma), \quad B r(t \rightarrow c Z)$ increase with the increasing of $m_{\tilde{b}}$ slowly, but $\tan \beta$ affects both of them obviously. And with the increasing of $\tan \beta$, they can reach $5 \times 10^{-7}, 4 \times 10^{-7}$ respectively. However, Fig. 3b shows that $\operatorname{Br}(t \rightarrow c g)$ has a sharp decrease when $\tan \beta=15$, and the turning point is around $1850 \mathrm{GeV}$. Due to the fact that the contributions from down type squarks to the branching ratio is cancelled by the contributions from charge Higgs boson at the turning point. In addition, from the mass matrix of down type squarks, we can see that the masses of down type squarks increase with the increasing of $\tan \beta$ or $m_{\tilde{b}}$, hence the turning point of $m_{\tilde{b}}$ 


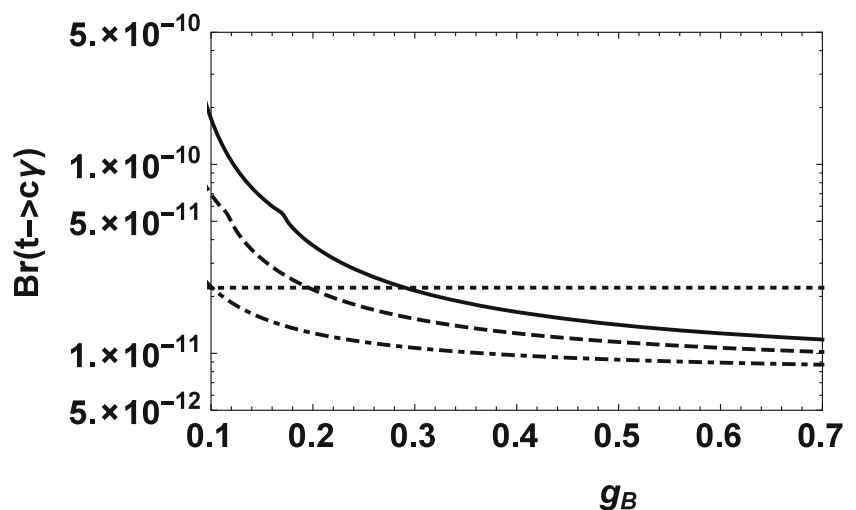

(a)

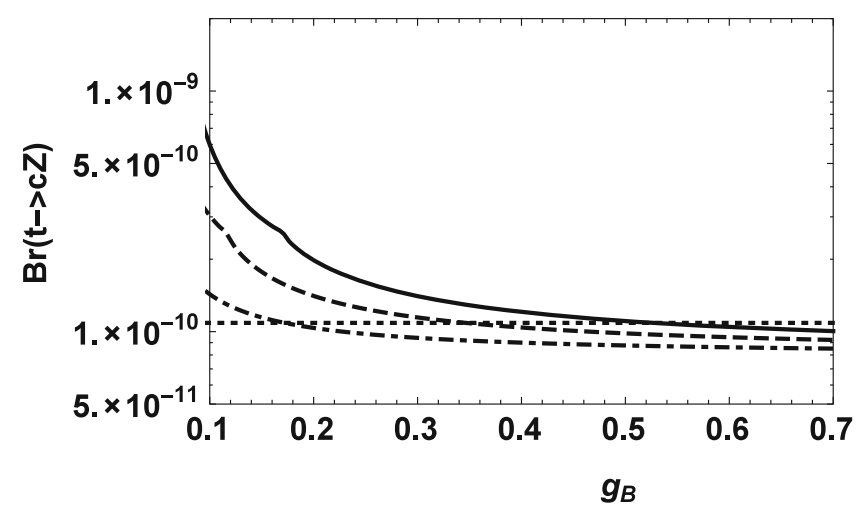

(c)

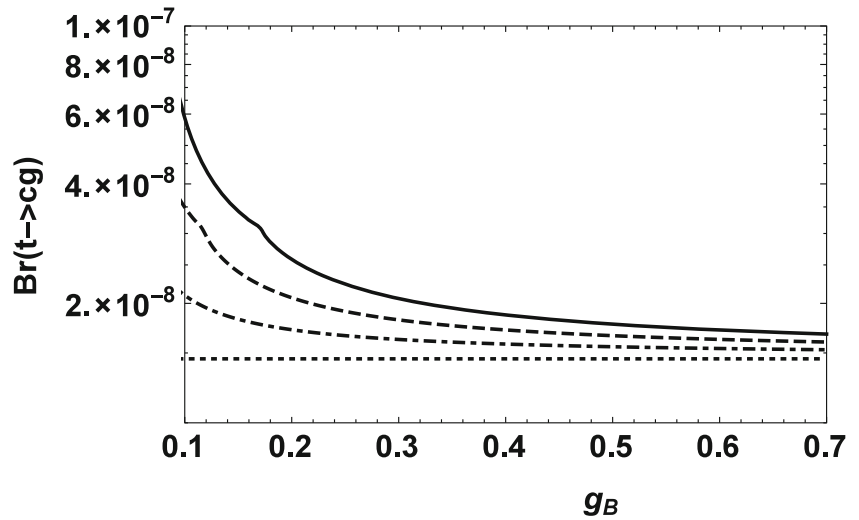

(b)

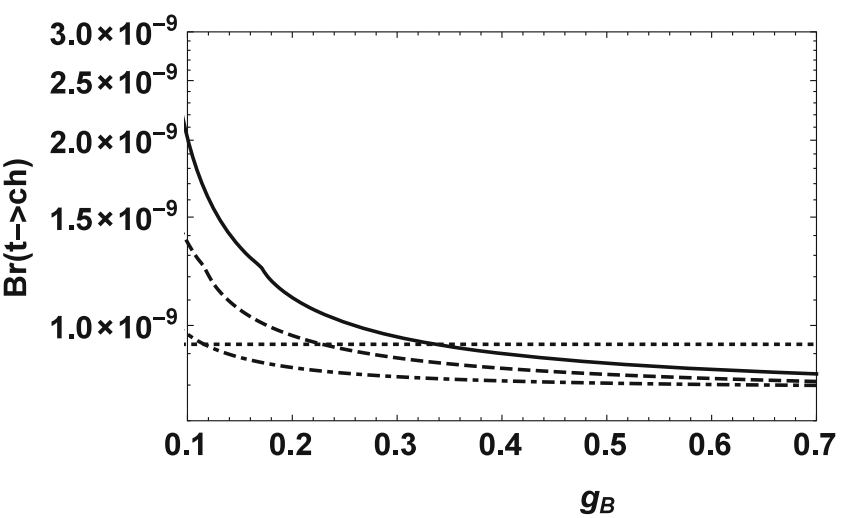

(d)

Fig. $4 \mathrm{Br}(t \rightarrow c \gamma)(\mathbf{a}), \operatorname{Br}(t \rightarrow c g)(\mathbf{b}), \operatorname{Br}(t \rightarrow c Z)(\mathbf{c}), \operatorname{Br}(t \rightarrow c h)(\mathbf{d})$ versus $g_{B}$ for $g_{Y B}=-0.6$ (solid line), $g_{Y B}=$ -0.4 (dashed line), $g_{Y B}=-0.2$ (dot-dashed line) are plotted. The dotted line denotes the MSSM predictions in the same parameter space

decreases with the increasing of $\tan \beta$, which results in the turning point less than $1700 \mathrm{GeV}$ when $\tan \beta=25,35$. The moving of turning point can be seen directly in Fig. 3d. The picture shows that the turning point decreases with the increasing of $\tan \beta$. In addition, with the increasing of $m_{\tilde{b}}$, the effect of $\tan \beta$ is more negligible to $\operatorname{Br}(t \rightarrow c h)$. Since the main contribution to these processes come from down type squarks, $m_{\tilde{b}}$ affects the numerical results mainly through influencing the masses of the third generation down type squarks. Meanwhile, $\tan \beta$ not only presents in the diagonal sector of the mass matrix, but also dominates the offdiagonal sector, which indicates that $\tan \beta$ affects the numerical results mainly through influencing the mass of the down type squarks and the corresponding rotation matrix in the couplings involve down type squarks.

In order to see how new coupling constants $g_{B}$ and $g_{Y B}$ in the B-LSSM affect $\operatorname{Br}(t \rightarrow c \gamma), \operatorname{Br}(t \rightarrow c g), B r(t \rightarrow c Z)$ and $\operatorname{Br}(t \rightarrow c h)$, we continue to fix $\tan \beta=4, \tan \beta^{\prime}=$ $1.2, m_{\tilde{b}}=1.5 \mathrm{TeV}, A_{t}=-2$. Considering the limits from B physics and concrete Higgs mass, the allowed region of $g_{B}$ and $g_{Y B}$ are

$$
-0.7<g_{Y B}<0, \quad 0.1<g_{B}<0.7 .
$$

Then we present $\operatorname{Br}(t \rightarrow c \gamma), \operatorname{Br}(t \rightarrow c g), \operatorname{Br}(t \rightarrow c Z)$ and $\operatorname{Br}(t \rightarrow c h)$ varying with $g_{B}$ in Fig. 4a-d respectively, where the three lines denote $g_{Y B}=-0.6$ (dotdashed line), $g_{Y B}=-0.4$ (dashed line) and $g_{Y B}=-0.2$ (dot-dashed line). In order to compare with the MSSM, we also plot the MSSM predictions in the same parameter space (dotted line). It can be noted that $\mathrm{Br}(t \rightarrow c g)$ in the B-LSSM can exceed the MSSM prediction easily in our chosen parameter space. And $\mathrm{Br}(t \rightarrow c \gamma), \operatorname{Br}(t \rightarrow c Z), \operatorname{Br}(t \rightarrow c h)$ in the BLSSM can exceed the MSSM predictions when $g_{B}$ is small and $\left|g_{Y B}\right|$ is large. In addition, as $g_{Y B}$ approach to zero, all of the branching ratios depend on $g_{B}$ negligibly, which indicates that the effect of $g_{B}$ to these four processes is influenced by the strength of gauge kinetic mixing strongly. $g_{B}$ and $g_{Y B}$ affect the numerical results mainly in three ways. Firstly, $g_{B}$ and $g_{Y B}$ affect $\mathrm{Br}(t \rightarrow c \gamma, g, Z, h)$ by influencing the down type squark masses and the corresponding rotation matrix, which appears in the couplings involve the down type squarks. Secondly, they make new contributions to $\mathrm{Br}(t \rightarrow$ 
$c Z$ ) by the $Z-Z^{\prime}$ mixing. Thirdly, they affect the theoretical prediction on $\operatorname{Br}(t \rightarrow c h)$ by mixing the Higgs doublets with the exotic singlets.

\section{Summary}

In the $U(1)_{B-L}$ extension of MSSM, under a minimal flavor violating assumption for the soft breaking terms, we focused on the top quark rare decay processes $t \rightarrow c \gamma, c g, c Z, c h$. Compared with the MSSM, new definition of the down type squark masses can affect the theoretical evaluation on these processes. In addition, the mixing in the scalar sector and $Z-Z^{\prime}$ sector can also make new contributions to $t \rightarrow c h$ and $t \rightarrow c Z$ decay channel respectively. In our used parameter space, the numerical results show that all of these processes are well below the experiment limits. And $\tan \beta$ is a major parameter to the processes $t \rightarrow c \gamma, c g, c Z, c h$, the corresponding branching ratios can be $5 \times 10^{-7}, 2 \times 10^{-6}$, $4 \times 10^{-7}, 3 \times 10^{-9}$ respectively. Simultaneously, new gauge coupling constants $g_{B}, g_{Y B}$ in the B-LSSM can also affect the numerical results of $\mathrm{Br}(t \rightarrow c \gamma, c g, c Z, c h)$.

Acknowledgements The work has been supported by the National Natural Science Foundation of China (NNSFC) with Grants No. 11535002, No. 11647120, and No. 11705045, Natural Science Foundation of Hebei province with Grants No. A2016201010 and No. A2016201069, Foundation of Department of Education of Liaoning province with Grant No. 2016TSPY10, Youth Foundation of the University of Science and Technology Liaoning with Grant No. 2016QN11, Hebei Key Lab of Optic-Eletronic Information and Materials, and the Midwest Universities Comprehensive Strength Promotion project.

Open Access This article is distributed under the terms of the Creative Commons Attribution 4.0 International License (http://creativecomm ons.org/licenses/by/4.0/), which permits unrestricted use, distribution, and reproduction in any medium, provided you give appropriate credit to the original author(s) and the source, provide a link to the Creative Commons license, and indicate if changes were made.

Funded by SCOAP ${ }^{3}$.

\section{References}

1. J.L. Diaz-Cruz, R. Martinez, M.A. Perez, A. Rosado, Phys. Rev. D 41, 891 (1990)

2. G. Eilam, J.L. Hewett, A. Soni, Phys. Rev. D 44, 1473 (1991)

3. B. Mele, S. Petrarca, A. Soddu, Phys. Lett. B 435, 401 (1998)

4. G. Eilam, J.L. Hewett, A. Soni, Phys. Rev. D 59, 039901(E) (1999)

5. J.A. Aguilar-Saavedra, B.M. Nobre, Phys. Lett. B 553, 251 (2003)

6. J.A. Aguilar-Saavedra, Acta Phys. Pol. 35, 2695 (2004)

7. F. Larios, R. Martinez, M.A. Perez, Int. J. Mod. Phys. A 21, 3473 (2006)

8. K. Agashe et al., [Top Quark Working Group]. arXiv:1311.2028 [hep-ph]

9. D. Bardhan, PoS CKM 2016, 131 (2017). arXiv:1705.08486 [hep$\mathrm{ph}]$

10. CMS Collab., J. High Energy Phys. 1604, 035 (2016)

11. CMS Collab., J. High Energy Phys. 1702, 028 (2017)
12. CMS Collab., Eur. Phys. J. C 76, 55 (2016)

13. CMS Collab., J. High Energy Phys. 1710, 129 (2017)

14. CMS Collab., J. High Energy Phys. 1702, 079 (2017)

15. CMS Collab., J. High Energy Phys. 1512, 061 (2015)

16. CMS Collab., CMS-PAS-TOP-17-003

17. CMS Collab., ATLAS-CONF-2017-070

18. CMS Collab., J. High Energy Phys. 1707, 003 (2017)

19. J.L. Diaz-Cruz, R. Martinez, M.A. Perez, A. Rosado, Phys. Rev. D 41, 891 (1900)

20. B. Grzadkowski, J.F. Gunion, P. Krawczyk, Phys. Lett. B 268, 106$11(1991)$

21. A. Arhrib, Phys. Lett. B 612, 263 (2005)

22. C.S. Li, R.J. Oakes, J.M. Yang, Phys. Rev. D 49, 293 (1994)

23. J. Guasch, J. Sola, Nucl. Phys. B 562, 3 (1999). arXiv:hep-ph/9906268

24. K. Suxho, PoS PLANCK 2015, 128 (2015). arXiv:1512.08661 [hep-ph]

25. J.M. Yang, B.L. Young, X. Zhang, Phys. Rev. D 58, 055001 (1998)

26. G. Eilam, A. Gemintern, T. Han, J.M. Yang, X. Zhang, Phys. Lett. B 510, 227 (2001)

27. G. Lu, F. Yin, X. Wang, L. Wan, Phys. Rev. D 68, 015002 (2003). arXiv:hep-ph/0303122

28. K. Agashe, G. Perez, A. Soni, Phys. Rev. D 75, 015002 (2007). arXiv:hep-ph/0606293

29. M. Ambroso, B.A. Ovrut, Int. J. Mod. Phys. A 26, 1569 (2011). arXiv:1005.5392 [hep-th]

30. P.F. Perez, S. Spinner, Phys. Rev. D 83, 035004 (2011)

31. W. Abdallah, A. Hammad, S. Khalil, S. Moretti, Phys. Rev. D 95(5), 055019 (2017). arXiv:1608.07500 [hep-ph]

32. S. Khalil, H. Okada, T. Toma, JHEP 1107, 026 (2011). arXiv:1102.4249 [hep-ph]

33. A. Elsayed, S. Khalil, S. Moretti, Phys. Lett. B 715, 208 (2012). arXiv:1106.2130 [hep-ph]

34. G. Brooijmans et al. arXiv:1203.1488 [hep-ph]

35. L. Basso, F. Staub, Phys. Rev. D 87, 015011 (2013). arXiv: 1210.7946 [hep-ph]

36. L. Basso et al., Comput. Phys. Commun. 184, 698 (2013). arXiv: 1206.4563 [hep-ph]

37. A. Elsayed, S. Khalil, S. Moretti, A. Moursy, Phys. Rev. D 87, 053010 (2013). arXiv:1211.0644 [hep-ph]

38. S. Khalil, S. Moretti, Rept.Prog.Phys. 80 (2017). arXiv: 1503.08162 [hep-ph]

39. C.S. Aulakh, A. Melfo, A. Rasin, G. Senjanovic, Phys. Lett. B 459 , 557 (1999). arXiv:hep-ph/9902409

40. S. Khalil, A. Masiero, Phys. Lett. B 665, 374 (2008). arXiv:0710.3525 [hep-ph]

41. T.R. Dulaney, P. Fileviez Perez, M.B. Wise, Phys. Rev. D 83, 023520 (2011). arXiv:1005.0617 [hep-ph]

42. V. Barger, P. Fileviez Perez, S. Spinner, Phys. Rev. Lett. 102, 181802 (2009). arXiv:0812.3661 [hep-ph]

43. J. Pelto, I. Vilja, H. Virtanen, Phys. Rev. D 83, 055001 (2011). arXiv: 1012.3288 [hep-ph]

44. K.S. Babu, Y. Meng, Z. Tavartkiladze, Phys. Lett. B 681, 37 (2009). arXiv:0901.1044 [hep-ph]

45. S. Khalil, H. Okada, Phys. Rev. D 79, 083510 (2009). arXiv:0810.4573 [hep-ph]

46. L. Basso, B. O'Leary, W. Porod, F. Staub, JHEP 1209, 054 (2012). arXiv:1207.0507 [hep-ph]

47. L. Delle Rose, S. Khalil, S.J.D. King, C. Marzo, S. Moretti, C.S. Un, Phys. Rev. D 96, 055004 (2017). arXiv:1702.01808 [hep-ph]

48. L. Delle Rose, S. Khalil, S. J. D. King, S. Kulkarni, C. Marzo, S. Moretti, C. S. Un. arXiv:1712.05232 [hep-ph]

49. B. O'Leary, W. Porod, F. Staub, JHEP 1205, 042 (2012). arXiv:1112.4600 [hep-ph]

50. W. Abdallah, S. Khalil, S. Moretti, Phys. Rev. D 91, 014001 (2015). arXiv:1409.7837 [hep-ph] 
51. L. Basso, Adv. High Energy Phys. 2015, Article ID 980687

52. S. Khalil, C.S. Un, Phys. Lett. B 763, 164 (2016). arXiv:1509.05391 [hep-ph]

53. A. Hammad, S. Khalil, S. Moretti, Phys. Rev. D 93(11), 115035 (2016). arXiv:1601.07934 [hep-ph]

54. F. Staub. arXiv:0806.0538 ]

55. F. Staub, Comput. Phys. Commun 181, 1077-1086 (2010). arXiv:0909.2863

56. F. Staub, Comput. Phys. Commun. 182, 808-833 (2011). arXiv: 1002.0840

57. F. Staub, Comput. Phys. Commun. 184, 1792-1809 (2013). arXiv:1207.0906

58. F. Staub, Comput. Phys. Commun. 185, 1773-1790 (2014). arXiv: 1309.7223

59. B. Holdom, Phys. Lett. B 166, 196 (1986)

60. T. Matsuoka, D. Suematsu, Prog. Theor. Phys. 76, 901 (1986)

61. F. del Aguila, G.D. Coughlan, M. Quiros, Nucl. Phys. B 307, 633 (1988)

62. F. del Aguila, J.A. Gonzalez, M. Quiros, Nucl. Phys. B 307, 571 (1988)

63. F. del Aguila, G.D. Coughlan, M. Quiros, Nucl. Phys. B 312, 751 (1989)

64. R. Foot, X.G. He, Phys. Lett. B 267, 509 (1991)

65. K.S. Babu, C.F. Kolda, J. March-Russell, Phys. Rev. D 57, 6788 (1998). arXiv:hep-ph/9710441

66. R. Fonseca, M. Malinsky, W. Porod, F. Staub, Nucl. Phys. B 854, 28-53 (2012). arXiv:1107.2670 [hep-ph]

67. P.H. Chankowski, S. Pokorski, J. Wagner, Eur. Phys. J. C 47, 187205 (2006)

68. R. Barate et al., ALEPH Collaboration. Eur. Phys. J. C 12, 183 (2000). arXiv:hep-ex/9904011

69. P. Abreu et al., DELPHI Collaboration. Phys. Lett. B 485, 45 (2000). arXiv:hep-ex/0103025
70. R. Fonseca, M. Malinsky, W. Porod, F. Staub, Nucl. Phys. B 854, 28-53 (2012)

71. M. Carena, J.R. Espinosaos, C.E.M. Wagner, M. Quir, Phys. Lett. B 355, 209 (1995)

72. M. Carena, M. Quiros, C.E.M. Wagner, Nucl. Phys. B 461, 407 (1996)

73. M. Carena, S. Gori, N.R. Shah, C.E.M. Wagner, JHEP 03, 014 (2012)

74. A. Denner, Fortsch. Phys. 41, 307 (1993). arXiv:0709.1075 [hep$\mathrm{ph}]$

75. C. Partignani et al. (PDG Collaboration), Chin. Phys. C 40, 100001(2016) and 2017 update

76. V. Shtabovenko, R. Mertig, F. Orellana, Comput. Phys. Commun. 207, 432 (2016). arXiv:1601.01167 [hep-ph]

77. R. Mertig, M. Bohm, A. Denner, Comput. Phys. Commun. 64, 345 (1991)

78. ATLAS Collab., ATLAS-CONF-2016-045

79. G. Cacciapaglia, C. Csaki, G. Marandella, A. Strumia, Phys. Rev. D 74, 033011 (2006). arXiv:hep-ph/0604111

80. M. Carena, A. Daleo, B.A. Dobrescu, T.M.P. Tait, Phys. Rev. D 70, 093009 (2004). arXiv:hep-ph/0408098

81. K.A. Olive et al., Particle Date Group. Chin. Phys. C 38, 090001 (2014)

82. ATLAS Collab., Phys. Rev. D 87, 012008 (2013)

83. CMS Collab., JHEP 1210, 018 (2012)

84. C.S. Un, O. Ozdal, Phys. Rev. D 93, 055024 (2016). arXiv:1601.02494 [hep-ph]

85. J. L. Yang, T. F. Feng, H. B. Zhang, R. F. Zhu, S. M. Zhao. arXiv:1803.09904 [hep-ph]

86. G. Aad et al., ATLAS Collaboration. Phys. Lett. B 716, 1 (2012) 